\title{
Prioritization of orphan disease-causing genes using topological feature and GO similarity between proteins in interaction networks
}

\author{
LI Min ${ }^{1}$, LI Qi ${ }^{1}$, GANEGODA Gamage Upeksha ${ }^{1}$, WANG JianXin ${ }^{1 *}$, WU FangXiang ${ }^{1,2}$ \\ \& PAN $\mathrm{Yi}^{1,3}$
}

\author{
${ }^{1}$ School of Information Science and Engineering, Central South University, Changsha 410083, China; \\ ${ }^{2}$ College of Engineering, University of Saskatchewan, Saskatoon, SK STN 5A9, Canada; \\ ${ }^{3}$ Department of Computer Science, Georgia State University, Atlanta, GA 30302-4110, USA
}

Received May 23, 2014; accepted July 15, 2014; published online October 14, 2014

\begin{abstract}
Identification of disease-causing genes among a large number of candidates is a fundamental challenge in human disease studies. However, it is still time-consuming and laborious to determine the real disease-causing genes by biological experiments. With the advances of the high-throughput techniques, a large number of protein-protein interactions have been produced. Therefore, to address this issue, several methods based on protein interaction network have been proposed. In this paper, we propose a shortest path-based algorithm, named SPranker, to prioritize disease-causing genes in protein interaction networks. Considering the fact that diseases with similar phenotypes are generally caused by functionally related genes, we further propose an improved algorithm SPGOranker by integrating the semantic similarity of gene ontology (GO) annotations. SPGOranker not only considers the topological similarity between protein pairs in a protein interaction network but also takes their functional similarity into account. The proposed algorithms SPranker and SPGOranker were applied to 1598 known orphan disease-causing genes from 172 orphan diseases and compared with three state-of-the-art approaches, ICN, VS and RWR. The experimental results show that SPranker and SPGOranker outperform ICN, VS, and RWR for the prioritization of orphan disease-causing genes. Importantly, for the case study of severe combined immunodeficiency, SPranker and SPGOranker predict several novel causal genes.
\end{abstract}

disease-causing genes, prioritization, gene ontology, protein interaction network, shortest path

Citation: Li M, Li Q, Ganegoda GU, Wang JX, Wu FX, Pan Y. Prioritization of orphan disease-causing genes using topological feature and GO similarity between proteins in interaction networks. Sci China Life Sci, 2014, 57: 1064-1071, doi: 10.1007/s11427-014-4747-6

An orphan disease (OD) is the disease that affects only a small percentage of the population, which is defined as a disease that affects fewer than 200000 inhabitants in the USA [1]. There are about 8000 ODs and most of which are genetic, and thus are present throughout the person's entire life, even if symptoms do not immediately appear [2,3]. More important, most ODs affect children at a very early

*Corresponding author (email: jxwang @ csu.edu.cn) age and about $30 \%$ of children with ODs will die before reaching their fifth birthday.

To find disease-causing genes (or genes involved in a biological process), researchers usually use the traditional positional cloning approaches or high-throughput genomic technologies to identify hundreds or even thousands of candidate genes [4]. However, it is too time-consuming and laborious to validate these candidate genes one by one through biological experiments [5,6]. Fortunately, the hu- 
man genome project has been completed and this project has achieved great success, at the same time, the development of high-throughput approaches has provided a large number of protein-protein interactions, which make it possible for us to study life activity at the network level [7-9]. Many network-based methods have been proposed to predict protein functions, identify essential proteins, and detect disease-causing genes and related complexes or pathways [10-21]. It also has been shown that genes associated with the similar phenotypes tend to share the common molecular signatures including similar expression profiles, participation in the same biological processes, pathways, or complexes [22-24]. Moreover, the research of human disease and the experiment of model organisms show that direct and indirect interactions occurred between protein pairs may be responsible for similar disease phenotypes [25-27]. These researches motivate us to predict disease-causing genes by using protein interaction networks.

In recent years, many network-based computational approaches have been proposed for prioritizing candidate disease genes [28-36]. Generally, the prioritization approaches can be grouped into two main categories: (i) global methods which model the information flow in the cell to assess the proximity and connectivity between known disease genes and candidate genes, such as PRIoritizatioN and Complex Elucidation (PRINCE [32]), Random Walk with Restart (RWR [33]), and PageRank with Priors (PRP [34]), and (ii) localized methods which predict new disease candidates by counting the direct interacting genes or computing the shortest paths between known disease genes and candidate genes, such as Interconnectedness (ICN [35]), and Vertex similarity-based frameworks (VS [36]).

In addition, some typical graph partitioning methods and clustering approaches, such as GS [37], MCL [38], VI-Cut [39], IPCA [40], MSCF [41], HC-PIN [42], and their improved algorithms, can also be used to discover candidate disease-related genes.

Although great progress has been made on the network-based methods, it is still a challenging task to identify disease-causing genes based on protein interaction network, for there are still a large number of false-positives or negatives existing in the current available protein-protein interaction data [43]. To reduce the effect of the false positives, different types of biological data related to proteins, such as gene expression profiles [44-48], orthology information [49], gene ontology (GO) annotations, have been used in the identification of protein complexes, discovery of essential proteins, prediction of protein functions, or the detection of disease-causing genes.

Based on an overall analysis of 'guilt-by-association' principle and the effectiveness of local network information, we proposed a localized algorithm, SPranker, to prioritize ODs-causing genes. To reduce the effect of the false positives in protein interaction network, we further integrated
GO annotations to improve our proposed algorithm SPranker. The improved algorithm, named SPGOranker, prioritizes the disease-causing candidates by combining GO similarity of the candidate genes with their topological similarity. The proposed algorithms SPranker and SPGOranker were tested on the 172 ODs with at least five known causal genes (from Orphanet database [50]). In our experiments, the leave-one-out cross-validation was used to validate the effectiveness of the proposed algorithms. Based on the leave-one-out cross-validation, the proposed algorithm SPranker was shown to outperform three other state-of-theart algorithms: RWR [33], ICN [35], and VS [36]. The combination of GO annotations contributed to the prioritization of disease-causing genes and SPGOranker was shown to perform better than SPranker. Moreover, SPranker and SPGOranker were applied to predict potential novel candidate genes by prioritization of the immediate neighbors of known OD genes in the human protein interaction network.

\section{Methods}

\subsection{SPranker: shortest path based algorithm to priori- tize disease-causing genes}

The common assumption of network-based disease-causing gene prioritization methods is that genes that are physically or functionally close to each other tend to be involved in the same biological pathways and have similar effects on phenotypes, which is known as 'guilt-by-association' principle [51]. Hence, the most important thing is how to measure the similarities between the known disease genes and candidate genes in a protein interaction network. In this paper, we use two different strategies to calculate the similarity between proteins: one is for the connected protein pairs and the other is for the proteins which do not have edges connecting them directly.

To describe the proposed algorithm easily, we first give some necessary definitions. A protein interaction network is described as an undirected graph $G=(V, E, W)$, where $V$ represents the set of proteins, an edge $\left(v_{i}, v_{j}\right) \in E$ denotes that the protein $v_{i}$ and the protein $v_{j}$ connect with each other in the network, and $\mathrm{w}\left(v_{i}, v_{j}\right) \in W$ represents the weight of the edge $\left(v_{i}, v_{j}\right)$. For an unweighted graph $G, \mathrm{w}\left(v_{i}, v_{j}\right)=1$ if and only if the protein $v_{i}$ and the protein $v_{j}$ connect each other in the graph $G$, otherwise $w\left(v_{i}, v_{j}\right)=0$. Given a protein $v \in \mathrm{V}$, its neighbors are the proteins which interact with it in the network. The set of neighbors of a protein $v$ is marked as $N_{v}$.

From the topological view, two proteins are considered to be similar if they have more common immediate neighbors in the network. Hence, for a pair of proteins $\left(v_{i}\right.$ and $v_{j}$ ) which connect each other in the protein interaction network, we calculate its similarity $\operatorname{Sim}^{t}\left(v_{i}, v_{j}\right)$ by using the following formula: 


$$
\operatorname{Sim}^{t}\left(v_{i}, v_{j}\right)=\frac{2 * w\left(v_{i}, v_{j}\right)+\sum_{v_{k} \in\left(N_{v_{i}} \cap N_{v_{j}}\right)} w\left(v_{i}, v_{k}\right) * w\left(v_{j}, v_{k}\right)}{\sqrt{\sum_{v_{k} \in N_{v_{i}}} w\left(v_{i}, v_{k}\right)^{2}} \times \sqrt{\sum_{v_{k} \in N_{v_{j}}} w\left(v_{j}, v_{k}\right)^{2}}},
$$

where $w\left(v_{i}, v_{j}\right)=1$ if the protein $v_{i}$ and the protein $v_{j}$ connect each other in the network.

For a pair of proteins $\left(v_{i}\right.$ and $\left.v_{j}\right)$ which do not have any edges connecting them in the graph $G$, their similarity, marked as $\operatorname{Sim}^{t^{*}}\left(v_{i}, v_{j}\right)$, is calculated by the following formula:

$$
\operatorname{Sim}^{t^{*}}\left(v_{i}, v_{j}\right)=\operatorname{Sim}^{t}\left(v_{i}, u_{1}\right) * \prod_{k=1}^{n-1} \operatorname{Sim}^{t}\left(u_{k}, u_{k+1}\right) * \operatorname{Sim}^{t}\left(u_{n}, v_{j}\right)
$$

where $u_{k}$ denotes the intermediate node on the shortest path from the protein $v_{i}$ to the protein $v_{j}$. If there exist more than one shortest path for a pair of nodes $v_{i}$ and $v_{j}$, all the shortest paths will be kept and the corresponding $\operatorname{Sim}^{t^{* *}}\left(v_{i}, v_{j}\right)$ will be calculated and the largest $\operatorname{Sim}^{t^{*}}\left(v_{i}, v_{j}\right)$ will be kept. A schematic drawing of a shortest path from $v_{i}$ to $v_{j}$ with $n+1$ hops is shown in Figure 1.

Generally, the shortest path from a given protein $v_{i}$ to a target protein $v_{j}$ was calculated by computing its hops from $v_{i}$ to $v_{j}$ [36]. However, a path with the least hops may not be the true path. For example, as shown in Figure 2, there are three paths from the protein $v_{i}$ to the protein $v_{j}$ : path $1\left(v_{i} ; v_{3}\right.$; $v_{j}$ ) with two hops, path $2\left(v_{i} ; v_{1} ; v_{2} ; v_{j}\right)$ with three hops, and path $3\left(v_{i} ; v_{4} ; v_{5} ; v_{6} ; v_{j}\right)$ with four hops. The weight in the figure denotes the reliability between two proteins. It will be path 1 if the shortest path is selected by hops. However, path 2 will be selected if we consider the reliability of the edges on the path. It is difficult to be reachable from $v_{i}$ to $v_{j}$ if path 1 with the unreliable edge (whose weight is 0.01 and which may be the false positive edge and not there actually) is selected.

Hence, in our approach, for a pair of proteins we try to find the most reliable path between them. That means we must find the path with the highest continued product of edge weight by considering the "bucket effect". To avoid enumeration of all the possible paths between $v_{i}$ and $v_{j}$, we change the problem for calculating the continued product of edge weight for each path into the problem for finding a shortest path between $v_{i}$ and $v_{j}$.

To calculate the shortest path between a pair of two proteins, we use $1 / \operatorname{Sim}^{t}\left(v_{i}, v_{j}\right)$ to describe the distance between two connected proteins $v_{i}$ and $v_{j}$ and reweight the edge $\left(v_{i}, v_{j}\right)$ in the graph $G$. The shortest path is calculated by using the Dijkstra's algorithm [52].

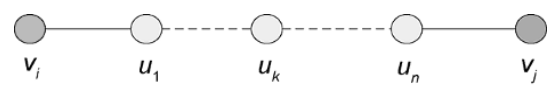

Figure 1 Schematic drawing of a shortest path from a protein $v_{i}$ to a protein $v_{j}$ with $n+1$ hops.

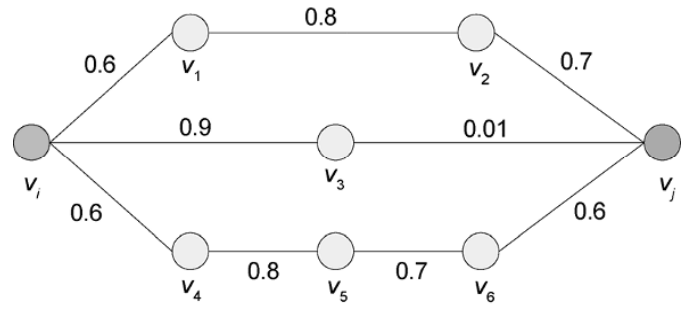

Figure 2 An example of shortest path calculation with multiple paths from a protein $v_{i}$ to a protein $v_{j}$.

Take Figure 2 for example, the lengths of path 1, path 2 and path 3 are $101.11(1 / 0.9+1 / 0.01), 4.35(1 / 0.6+1 / 0.8+$ $1 / 0.7)$, and $6.01(1 / 0.6+1 / 0.8+1 / 0.7+1 / 0.6)$, respectively. Hence, the shortest path between $v_{i}$ and $v_{j}$ is path 2 and it is the most reliable path among the three paths between $v_{i}$ and $v_{j}$ in Figure 2. Hence, the similarity of $v_{i}$ and $v_{j}$ is calculated by using path 2 not path 1 with the least hops.

For a given disease $d$, let $S_{d}$ denote the set of its known disease-causing genes. Then, for a new candidate gene $v_{i}$, the probability that it is also such a disease-causing gene is evaluated by the sum of similarities between it and the known disease-causing genes in $S_{d}$, as shown in eq. (3):

$$
\text { score }_{v_{i}}=\sum_{v_{j} \in S_{d}} \operatorname{Sim}^{t}\left(v_{i}, v_{j}\right) \text {. }
$$

All the candidate genes are then ranked based on these scores. The complexity of calculating a candidate gene's probability to be a disease-causing gene depends on the number of known disease-causing genes in $S_{d}$ and the complexity of computing the shortest paths.

\subsection{SPGOranker: integration of GO annotations into SPranker}

It has been shown that similarities among disorders imply involvement of functionally related gene products, which is generally summarized as "phenotypic overlap implies genetic overlap". Recent studies also showed that diseases with similar phenotypes often involve common molecular mechanisms and the functions of their corresponding disease-causing genes are generally similar.

Franke's work [53] shows that GO annotations are the most effective resources for the identification of disease-causing genes. Many other researchers also explored the relationship between the GO annotations and disease-causing genes and predicted new candidates by using their functional similarity to the known causal genes, such as G2D [54], POCUS [55], FP [56] and GFFST [57].

The Gene Ontology project [58] provides a controlled vocabulary of terms for describing gene product characteristics and gene product annotation data. The GO ontology is with a hierarchical structure and is generally described as a directed acyclic graph (DAG). There have been many com- 
putational methods for calculating the similarity between GO terms $[59,60]$. In this paper, we used one of our previous methods in [59] to calculate the GO term similarity by taking into account the hierarchical organization of functional annotations. For a given pair of proteins $v_{i}$ and $v_{j}$, their functional similarity $\operatorname{Sim}^{f}\left(v_{i}, v_{j}\right)$ is defined as the maximum similarity of their corresponding GO terms, as shown in eq. (4):

$$
\operatorname{Sim}^{f}\left(v_{i}, v_{j}\right)=\max _{c_{1 \in T_{i}} \cdot c_{2 \in T_{j}}}\left(\operatorname{goSim}\left(c_{1}, c_{2}\right)\right),
$$

where $T_{i}$ and $T_{j}$ are the corresponding sets of GO terms for the protein $v_{i}$ and the protein $v_{j}$, respectively. The functional similarity, marked as $\operatorname{goSim}\left(c_{1}, c_{2}\right)$, between the term $c_{1} \in T_{i}$ and term $c_{2} \in T_{j}$ is calculated by using the method in [59].

For each species, there are three types of annotations: biological processes, cellular components and molecular functions. In this paper, the molecular functions are used to calculate the functional similarity between proteins and an improved algorithm SPGOranker is proposed to prioritize disease-causing genes by integrating the functional similarity into SPranker. A parameter $\alpha$ is used to integrate the topological similarity and the functional similarity, as shown in eq. (5).

$$
\operatorname{Sim}\left(v_{i}, v_{j}\right)=a * \operatorname{Sim}^{t}\left(v_{i}, v_{j}\right)+(1-a) * \operatorname{Sim}^{f}\left(v_{i}, v_{j}\right) .
$$

The rest steps of SPGOranker are the same as those of SPranker. When $\alpha=1$, only the topological similarity is considered and SPGOranker will degenerate into SPranker.

\section{Results and discussion}

\subsection{Experimental data sources}

The human protein-protein interactions were downloaded from release 9 of the Human Protein Reference Database [61] with both redundant interactions and self-loops removed. Finally, a protein interaction network with 9763 genes and 37060 interactions was obtained and the unweighted network was used in our experiment.

The ODs and causal gene information were downloaded from Orphanet [50]. Thereafter, we merged some of the OD sub-types of a single disease based on the disorder names described in [30,33]. In our work, we selected 172 ODs that have at least five causal genes. The 172 ODs contain 1598 genes in total and 1063 OD-causing genes were found in the protein interaction network used in this paper.

\subsection{Cross-validation analysis}

To evaluate the performance of the proposed algorithms SPranker and SPGOranker for prioritizing disease-causing genes, we compared them with three state-of-the-art methods: RWR [33], ICN [35], and VS [36]. A leave-one-out cross-validation procedure was used to carry out the evaluation. In each cross validation trial, one causal gene with an OD ("target gene") from the data was removed, and each algorithm was evaluated by its success in assigning the rank to the "target gene". For each validation trial, one seed gene ("target gene") from 172 ODs was removed and mixed with 99 genes selected randomly from the protein interaction network to form a set of 100 candidate genes. The remaining seed genes are treated as the training set.

SPranker, SPGOranker, ICN, VS, and RWR were applied to the test set for prioritizing OD-causing genes. In this paper, the default value of $\alpha$ in SPGOranker is set to be 0.8. During each execution, the rank of the "target gene" was marked. The performance of each algorithm is evaluated in terms of success rate with the respective rank cut-off $(k)$. If the "target gene" is ranked among the top $k$ in a particular validation trial, it is considered as a "success". As what has been done in [36], we also used $k$ ranging from 1 to 30 in this paper. Validation trials are repeated until all the seed genes have been used as the target genes and their ranks are obtained. The "success rate" is defined as the ratio of successful validation trails and the total validation trails for all the existing OD genes from 172 ODs [36].

The results of the five algorithms SPranker, SPGOranker, ICN, VS, and RWR on the success rate with $k$ ranging from 1 to 30 are shown in Figure 3. When $k=1$, out of 1063 cases 407 are achieved successfully by SPGOranker. SPranker and VS also achieve similar performance with a success rate of $37.8 \%(402 / 1063)$ and $37.1 \%(394 / 1063)$. The success rates of RWR and ICN are $35.9 \%(381 / 1063)$ and $32.7 \%$ (348/1063), respectively. From Figure 3 we can see that SPGOranker performs best consistently with $k$ ranging from 1 to 30 , and SPranker also achieves a better result compared with ICN, VS, and RWR. These results imply the effectiveness of SPranker for the prioritization of OD-causing genes and the integration of GO annotations contributes to the improvement of detecting true OD-causing genes.

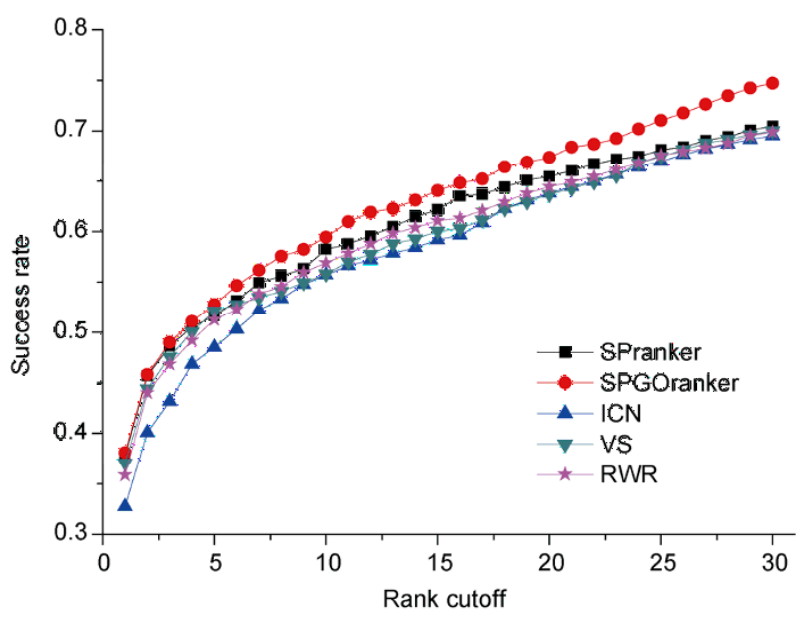

Figure 3 (color online) Comparison of five prioritization algorithms, SPranker, SPGOranker, ICN, VS, and RWR, on the success rate with $k$ ranging from 1 to 30 . 
To further investigate why and how SPranker and SPGOranker prioritize the disease-causing genes effectively, we analyze the intersection of disease-causing genes identified by the algorithms SPranker, SPGOranker, ICN, VS and RWR with $k=1$. The analysis results are shown in Table 1 .

As shown in Table 1, there are 323 disease-causing genes both identified by SPranker and by SPGOranker, which is about $80.3 \%$ of the true predictions by SPranker and by SPGOranker. Out of 394 disease-causing genes identified by VS, 309 genes (about 78.4\%) are also discovered by SPGOranker and SPGOranker predicts 96 different true disease-causing genes. ICN identifies 348 disease-causing genes and about $77.0 \%$ are covered by SPGOranker. One hundred and thirty-seven different true disease-causing genes are predicted by SPGOranker, which is about 1.7 times that identified by ICN. Out of 381 disease-causing genes predicted by RWR, 288 genes (about 75.6\%) are also discovered by SPGOranker and SPGOranker predicts 117 different true disease-causing genes.

\subsection{Analysis of the effect of parameter $\alpha$}

In the above analysis, $\alpha=0.8$ is used in SPGOranker. To analyze the effect of parameter $\alpha$ on the results, we change the value of $\alpha$ from 0.0 to 1.0 with 0.1 increments. When $\alpha=0.0$, only GO information is used to calculate the similarity between proteins. The analysis results are shown in Figure 4.

Table 1 Contingency table of disease-causing genes identified by the algorithms SPranker, SPGOranker, ICN, VS and RWR with $k=1$

\begin{tabular}{cccccc}
\hline & SPranker & SPGOranker & VS & ICN & RWR \\
\hline SPranker & 402 & 323 & 309 & 268 & 287 \\
SPGOranker & 323 & 405 & 309 & 268 & 288 \\
VS & 309 & 309 & 394 & 264 & 285 \\
ICN & 268 & 268 & 264 & 348 & 257 \\
RWR & 287 & 288 & 285 & 257 & 381 \\
\hline
\end{tabular}

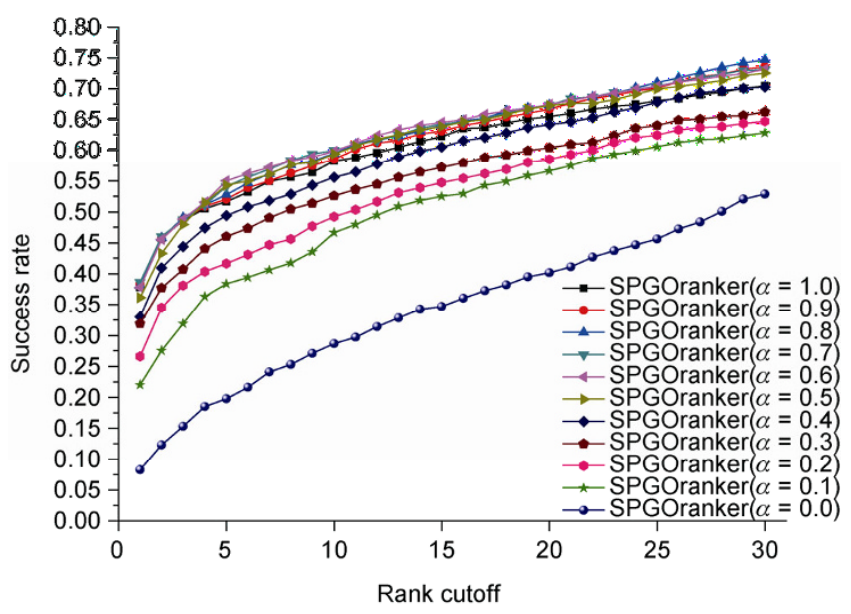

Figure 4 (color online) Cross validation results with different values of the parameter $\alpha$.
From Figure 4 we can see that with the increase of rank cutoff $k$, the success rates also increase. For the same rank cutoff $k$, the success rates of SPGOranker also increase with the increase of $\alpha$ up to 0.6 . For all the rank cutoff $k=5,10$, $15,20,25,30$, similar results are obtained by SPGOranker with $\alpha$ ranging from 0.6 to 0.8 . There is a small decline for the success rate when $\alpha>0.8$ is used. When $\alpha=1$, a more clear decline for the success rate can be seen from Figure 4 as SPGOranker has degenerated into SPranker. The analysis of parameter $\alpha$ 's effect shows that the topological similarity and the functional similarity have their own special contributions to the prioritization of disease-causing genes.

\subsection{Predicting novel causing genes of 10 ODs}

The proposed algorithms SPranker and SPGOranker were applied on 172 ODs to predict potential novel OD-causing genes. We select ten ODs which are associated with more than 10 known valid causing genes and have known protein-protein interactions for all of their causing genes. The known OD causing genes are considered as seeds and the immediate neighbors of the known causing genes in the protein interaction network are considered as candidate disease genes. Taken the severe combined immunodeficiency (SCID) as case study, the top 10 candidate genes predicted by SPranker, SPGOranker, ICN, VS, and RWR are shown in Table 2.

SCID is a genetic disorder characterized by the absence of functional T-lymphocytes. It is the most severe form of primary immunodeficiency. As shown in Table 2, the first predictions of SPranker and SPGOranker are both "ZAP70 (zeta-chain-associated protein $70 \mathrm{kD}$ )", which is also identified by VS as the top 1 . Though ZAP70 is not collected into Orphanet [50], recent research has shown that ZAP70 deficiency is an autosomal recessive form of severe combined immune deficiency (SCID). Moreover, Pagon's work [62] also shows that ZAP70 is signaling of abnormal $\mathrm{T}$ cell receptor, which is caused by cell-mediated immunodeficiency.

A new candidate gene "JAK1 (Janus kinase 1)" is identified as the second one both by SPranker and SPGOranker,

Table 2 Top 10 predictions of severe combined immunodeficiency

\begin{tabular}{cccccc}
\hline Rank & SPranker & SPGOranker & ICN & VS & RWR \\
\hline 1 & ZAP70 & ZAP70 & 1IL2RB & ZAP70 & STIM2 \\
2 & JAK1 & JAK1 & TRAT1 & JAK1 & IPO5 \\
3 & IL2RB & STAT5A & JAK1 & STAT5A & TRPC6 \\
4 & STAT5A & IL2RB & ZAP70 & TRB@ & TRPC3 \\
5 & CD3G & PTPN6 & PTPN22 & CD3G & XRCC4 \\
6 & TRB@ & CD3G & STAT5A & PTPN6 & LIG4 \\
7 & PTPN6 & TRB@ & PTPN6 & IL2RB & ACTB \\
8 & TRAT1 & PTPN22 & SHC1 & PTPN22 & GRB2 \\
9 & TRA@ & TRAT1 & SYK & TRA@ & THBS1 \\
10 & TSLP & IL7 & PIK3R1 & TRAT1 & FYN \\
\hline
\end{tabular}


Table 3 Ten ODs and their top 5 predictions ${ }^{\text {a) }}$

\begin{tabular}{|c|c|c|c|}
\hline Disease name & $\mathrm{KN}$ & Method & Top 5 predictions \\
\hline \multirow{2}{*}{ Retinitis pigmentosa } & \multirow{2}{*}{35} & SPranker & RDH5, DHX30, SLC24A1, PRPH, FIZ1 \\
\hline & & SPGOranker & RDH5, DHX30, SLC24A1, FIZ1, PRPH \\
\hline \multirow{2}{*}{ Microdeletion syndrome } & \multirow{2}{*}{21} & SPranker & FOXP4, FOXP1, SLC7A8, MEF2D, NKX2-5 \\
\hline & & SPGOranker & FOXP4, FOXP1, SLC7A8, MEF2D, NKX2-5 \\
\hline \multirow{2}{*}{ Cone rod dystrophy } & \multirow{2}{*}{17} & SPranker & CNGB1, CABP4, GUCA2B, NPHP4, ROM1 \\
\hline & & SPGOranker & CNGB1, CABP4, NPHP4, GUCA2B, ROM1 \\
\hline Severe combined immunodeficiency & 17 & SPGOranker & ZAP70, JAK1, STAT5A, IL2RB, PTPN6 \\
\hline \multirow{2}{*}{ Fanconi anemia } & \multirow{2}{*}{15} & SPranker & HES1, XRCC3, SAMD3, CYP19A1, RAD51 \\
\hline & & SPGOranker & HES1, XRCC3, RAD51, USP1, CYP19A1 \\
\hline \multirow{2}{*}{ Zellweger syndrome } & \multirow{2}{*}{14} & SPranker & PEX7, ABCD1, ABCD2, ABCD3, PXMP4 \\
\hline & & SPGOranker & ABCD1, ABCD2, PEX7, ABCD3, PEX11A \\
\hline \multirow{2}{*}{ Neonatal adrenoleukodystrophy } & \multirow{2}{*}{12} & SPranker & PEX7, ABCD1, ABCD2, ABCD3, PXMP4 \\
\hline & & SPGOranker & ABCD1, ABCD2, PEX7, ABCD3, PXMP4 \\
\hline Infantile Refsum disease & 12 & SPGOranker & ABCD1, ABCD2, PEX7, ABCD3, PXMP4 \\
\hline \multirow{2}{*}{ Papillary or follicular thyroid carcinoma } & \multirow{2}{*}{11} & SPranker & OCRL, TRIM28, RNF14, ZNF10, SHC1 \\
\hline & & SPGOranker & OCRL, TRIM28, RNF14, SHC1, GAB1 \\
\hline \multirow{2}{*}{ Romano-Ward syndrome } & \multirow{2}{*}{11} & SPranker & KCNE4, ALG10B, NDUFS6, ALG10, KCNJ3 \\
\hline & & SPGOranker & KCNE4, ALG10B, KCNJ3, NDUFS6, ALG10 \\
\hline
\end{tabular}

a) $\mathrm{KN}$ means the number of the known disease-causing genes in the corresponding OD.

and "IL2RB" is listed as the third one by SPranker and the fourth one by SPGOranker. Russell's study [63] shows that gene JAK1 and gene IL-2R $\beta \& \gamma$ interact with each other, and $\mathrm{C}$ chain mutations lead to X-linked severe combined immunodeficiency. In addition, "TSLP (thymic stromal lymphocytes)" was listed as the 10th one by SPranker, but did not appear at any top 10 list by any other four algorithms. It has been shown that TSLP plays a role in controlling innate and adaptive immune responses [64].

The top 5 predictions for each OD by SPranker and SPGOranker are shown in Table 3.

\section{Conclusion}

In this study, we proposed two algorithms SPranker and SPGOranker to prioritize disease-causing genes in protein interaction networks. SPranker is a simple localized algorithm which only uses a protein interaction network. SPGOranker prioritizes candidates by considering both topological similarity and functional similarity between the predicted candidates and the known disease-causing genes. The proposed algorithms SPranker and SPGOranker were applied to 1598 known orphan disease-causing genes (ODGs) from 172 orphan diseases (ODs) and compared with three state-of-the-art approaches, ICN, VS and RWR. The experimental results show that SPranker and SPGOranker outperform ICN, VS, and RWR for the prioritization of orphan disease-causing genes. Importantly, the top predictions of SPranker and SPGOranker for the severe combined immunodeficiency match the known literature, providing further investigation of several novel causal relationships.

It is important to note that the performance of the proposed algorithm SPranker will rely on the quality of the protein interaction network though the only usage of network makes it simple and convenient to be used. For the algorithm SPGOranker, the combination of GO annotations improves its performance for the prioritization of disease-causing genes, but takes extra time to compute the GO term similarity and makes it more complex. Of course, the extra time costs are worthwhile. Moreover, only the molecular functions are considered in this paper. In our future work, the biological processes, cellular components and other biological information will be further considered.

This work was supported in part by the National Natural Science Foundation of China (61370024, 61428209, 61232001) and Program for New Century Excellent Talents in University (NCET-12-0547).

1 Dear JW, Lilitkarntakul P, Webb DJ. Are rare diseases still orphans or happily adopted? The challenges of developing and using orphan medicinal products. British J Clin Pharmacol, 2006, 62: 264-271

2 Schieppati AHJ, Daina E, Aperia A. Why rare diseases are an important medical and social issue. Lancet, 2008, 371: 2039-2041

3 Stolk P, Willemen MJC, Leufkens HGM. Rare essentials: drugs for rare diseases as essential medicines. Bull World Health Org, 2006, 84: 745-751

4 Botstein D, Risch N. Discovering genotypes underlying human phenotypes: past successes for mendelian disease, future approaches for complex disease. Nat Genet, 2003, 33: 228-237

5 Glazier AM, Nadeau JH, Aitman TJ. Finding genes that underlie complex traits. Science, 2002, 298: 2345-2349

6 McCarthy MI, Abecasis GR, Cardon LR, Goldstein DB, Little J, Ioannidis JP, Hirschhorn JN. Genome-wide association studies for 
complex traits: consensus, uncertainty and challenges. Nat Rev Genet, 2008, 9: 356-369

7 Wang J, Li M, Deng Y, Pan Y. Recent advances in clustering methods for protein interaction networks. BMC Genomics, 2010, 11: S10

8 Li M, Wu X, Wang J, Pan Y. Towards the identification of protein complexes and functional modules by integrating PPI network and gene expression data. BMC Bioinformatics, 2012, 13: 109

9 Zhao B, Wang J, Li M, Wu FX. Detecting protein complexes based on uncertain graph model. IEEE/ACM Trans Comput Biol Bioinform, 2014, doi:10.1109/TCBB.2013.2297915

10 Zhong J, Wang J, Peng W, Zhang Z, Pan Y. Prediction of essential proteins based on gene expression programming. BMC Genomics, 2013, 14: 1-8

11 Wang J, Peng W, Wu FX. Computational approaches to predicting essential proteins: a survey. Proteomics Clin Appl, 2013, 7: 181-192

12 Peng W, Wang J, Cai J, Chen L, Li M, Wu FX. Improving protein function prediction using domain and protein complexes in PPI networks. BMC Syst Biol, 2014, 8: 35

13 Wang J, Ren J, Li M, Wu FX. Identification of hierarchical and overlapping functional modules in PPI networks. IEEE Trans NanoBiosci, 2012, 11: 386-393

14 Wang J, Liu B, Li M and Pan Y. Identifying protein complexes from interaction networks based on clique percolation and distance restraction. BMC Genomics, 11: S10

15 Li M, Wang J, Chen J, Cai Z, Chen G. Identifying the overlapping complexes in protein interaction networks. Int J Data Min Bioinform, 2010, 4: 91-108

16 Peng W, Wang J, Cheng Y, Lu Y, Wu FX, Pan Y. UDoNC: an algorithm for identifying essential proteins based on protein domains and protein-protein interaction networks. IEEE/ACM Trans Comput Biol Bioinform, 2014, doi: 10.1109/TCBB.2014.2338317

17 Zhao B, Wang J, Li M, Wu FX, Pan Y. Prediction of essential proteins based on overlapping essential modules. IEEE Trans NanoBiosci, 2014, doi: 10.1109/TNB.2014.2337912

$18 \mathrm{Li} \mathrm{M}$, Wang J, Wang H, Pan Y. Identification of essential proteins from weighted protein interaction networks. J Bioinform Comput Biol, 2013, 11: 1341002

19 Wang J, Li M, Wang H, Pan Y. Identification of essential proteins based on edge clustering coefficient. IEEE/ACM Trans Comput Biol Bioinform, 2012, 9: 1070-1080

20 Li M, Zhang H, Wang J, Pan Y. A new essential protein discovery method based on the integration of protein-protein interaction and gene expression data. BMC Syst Biol, 2012, 6: 15

21 Li M, Wang J, Chen X, Wang H, Pan Y. A local average connectivity-based method for identifying essential proteins from the network level. Comput Biol Chem, 2011, 35: 143-150

22 Barabási AL, Gulbahce N, Loscalzo J. Network medicine: a network-based approach to human disease. Nat Rev Genet, 2011, 12: $56-68$

23 Goh KI, Cusick ME, Valle D, Childs B, Vidal M, Barabási AL. The human disease network. Proc Natl Acad Sci USA, 2007, 104: $8685-8690$

24 Feldman I, Rzhetsky A, Vitkup D. Network properties of genes harboring inherited disease mutations. Proc Natl Acad Sci USA, 2008, 105: 4323-4328

25 Oti M, Brunner HG. The modular nature of genetic diseases. Clin Genet, 2007, 71: 1-11

26 Wood LD, Parsons DW, Jones S, Lin J, Sjöblom T, Leary RJ, Shen D, Boca SM, Barber T, Ptak J, Silliman N, Szabo S, Dezso Z, Ustyanksky V, Nikolskaya T, Nikolsky Y, Karchin R, Wilson PA, Kaminker JS, Zhang Z, Croshaw R, Willis J, Dawson D, Shipitsin M, Willson JK, Sukumar S, Polyak K, Park BH, Pethiyagoda CL, Pant PV, Ballinger DG, Sparks AB, Hartigan J, Smith DR, Suh E, Papadopoulos N, Buckhaults P, Markowitz SD, Parmigiani G, Kinzler KW, Velculescu VE, Vogelstein B. The genomic landscapes of human breast and colorectal cancers. Science, 2007, 318: 1108-1113

27 Lim J, Hao T, Shaw C, Patel AJ, Szabó G, Rual JF, Fisk CJ, Li N, Smolyar A, Hill DE, Barabási AL, Vidal M, Zoghbi HY. A protein-protein interaction network for human inherited ataxias and dis- orders of Purkinje cell degeneration. Cell, 2006, 125: 801-814

28 Navlakha S, Kingsford C. The power of protein interaction networks for associating genes with diseases. Bioinformatics, 2010, 26: $1057-1063$

29 Ganegoda GU, Wang J, Wu FX, Li M. Prediction of disease genes using tissue-specified gene-gene network. BMC Syst Biol, 2014, 8(Suppl 3): S3

30 Wang J, Chen G, Li M, Pan Y. Integration of breast cancer gene signatures based on graph centrality. BMC Syst Biol, 2011, 5: S10

31 Chen B, Wang J, Li M, Wu FX. Identifying disease causing genes by integrating multiple data sources. BMC Med Genom, 2014, 7(Suppl 2): $\mathrm{S} 2$

32 Vanunu O, Magger O, Ruppin E, Shlomi T, Sharan R. Associating genes and protein complexes with disease via network propagation. PLoS Comput Biol, 2010, 6: e1000641

33 Köhler S, Bauer S, Horn D, Robinson PN. Walking the interactome for prioritization of candidate disease genes. Am J Hum Genet, 2008, 82: 949-958

34 Chen J, Aronow BJ, Jegga AG. Disease candidate gene identification and prioritization using protein interaction networks. BMC Bioinformatics, 2009, 10: 73

35 Hsu CL, Huang YH, Hsu CT, Yang UC. Prioritizing disease candidate genes by a gene interconnectedness-based approach. BMC Genomics, 2011, 12: S25

36 Zhu C, Kushwaha A, Berman K, Jegga AG. A vertex similarity-based framework to discover and rank orphan disease-related genes. BMC Syst Biol, 2012, 6: S8

37 Navlakha S, Rastogi R, Shrivastava N. Graph summarization with bounded error. In: Proceedings of the 2008 ACM SIGMOD International Conference on Management of Data, 2008. 419-432

38 van Dongen S. Graph clustering via a discrete uncoupling process. SIAM J Matrix Anal Appl, 2008, 30: 121-141

39 Navlakha S, White J, Nagarajan N, Pop M, Kingsford C. Finding biologically accurate clusterings in hierarchical tree decompositions using the variation of information. J Computat Biol, 2010, 17: 503-516

40 Li M, Chen J, Wang J, Hu B, Chen G. Modifying the DPClus algorithm for identifying protein complexes based on new topological structures. BMC Bioinformatics, 2008, 9: 398

41 Ding X, Wang W, Peng X, Wang J. Mining protein complexes from PPI networks using the minimum vertex cut. Tsinghua Sci Technol, 2012, 17: 674-681

42 Wang J, Li M, Chen J, Pan Y. A fast hierarchical clustering algorithm for functional modules discovery in protein interaction networks. IEEE/ACM Trans Computat Biol Bioinform, 2011, 8: 607-620

43 Montanez G, Cho YR. Predicting false positives of protein-protein interaction data by semantic similarity measures. Curr Bioinform, 2013, 8: 339-346

44 Li M, Zheng R, Zhang H, Wang J, Pan Y. Effective identification of essential proteins based on priori knowledge, network topology and gene expressions. Methods, 2014, 67: 325-333

45 Tang X, Wang J, Zhong J, Pan Y. Predicting essential proteins based on weighted degree centrality. IEEE/ACM Trans Comput Biol Bioinform, 2014, 11: 407-418

46 Wang J, Peng X, Peng W, Wu FX. Dynamic protein interaction network construction and applications. Proteomics, 2014, 8: 338-352

47 Wang J, Peng X, Li M, Pan Y. Construction and application of dynamic protein interaction network based on time course gene expression data. Proteomics, 2013, 13: 301-312

48 Tang X, Feng Q, Wang J, He Y, Pan Y. Clustering based on multiple biological information: approach for predicting protein complexes. IET Syst Biol, 2013, 7: 223-230

49 Peng W, Wang J, Wang W, Liu Q, Wu FX, Pan Y. Iteration method for predicting essential proteins based on orthology and protein-protein interaction networks. BMC Syst Biol, 2012, 6: 87

50 Aymé S. Orphanet, an information site on rare diseases. Soins; la revue de référence infirmière, 2003, 672: 46

51 Wolfe CJ, Kohane IS, Butte AJ. Systematic survey reveals general applicability of guilt-by-association within gene coexpression net- 
works. BMC Bioinformatics, 2005, 6: 227

52 Dijkstra EW. A note on two problems in connexion with graphs. Numerische Mathematik, 1959, 1: 269-271

53 Franke L, van Bakel H, Fokkens L, de Jong ED, Egmont-Petersen M, Wijmenga C. Reconstruction of a functional human gene network, with an application for prioritizing positional candidate genes. Am J Hum Genet, 2006, 78: 1011-1025

54 Perez-Iratxeta C, Bork P, Andrade MA. Association of genes to genetically inherited diseases using data mining. Nat Genet, 2002, 31: 316-319

55 Turner FS, Clutterbuck DR, Semple CAM. POCUS: mining genomic sequence annotation to predict disease genes. Genome Biol, 2003, 4: R75-R75

56 Freudenberg J, Propping P. A similarity-based method for genome-wide prediction of disease-relevant human genes. Bioinformatics, 2002, 18: S110-115

57 Zhang P, Zhang J, Sheng H, Russo JJ, Osborne B, Buetow K. Gene functional similarity search tool (GFSST). BMC Bioinformatics, 2006, 7: 135

58 Ashburner M, Ball CA, Blake JA, Botstein D, Butler H, Cherry JM, Davis AP, Dolinski K, Dwight SS, Eppig JT, Harris MA, Hill DP, Issel-Tarver L, Kasarskis A, Lewis S, Matese JC, Richardson JE, Ringwald M, Rubin GM, Sherlock G. Gene Ontology: tool for the unification of biology. Nat Genet, 2000, 25: 25-29

59 Li M, Wu X, Pan Y, Wang J. hF-measure: a new measurement for evaluating clusters in protein-protein interaction networks. Prote- omics, 2013, 13: 291-300

60 Wang J, Dai L, Li M. GO semantic similarity-based false positive reduction of protein-protein interactions. In: IEEE International Joint Conference on Bioinformatics, Systems Biology and Intelligent Computing, 2009. 211-214

61 Peri S, Navarro JD, Kristiansen TZ, Amanchy R, Surendranath V, Muthusamy B, Gandhi TK, Chandrika KN, Deshpande N, Suresh S, Rashmi BP, Shanker K, Padma N, Niranjan V, Harsha HC, Talreja N, Vrushabendra BM, Ramya MA, Yatish AJ, Joy M, Shivashankar HN, Kavitha MP, Menezes M, Choudhury DR, Ghosh N, Saravana R, Chandran S, Mohan S, Jonnalagadda CK, Prasad CK, Kumar-Sinha C, Deshpande KS, Pandey A. Human protein reference database as a discovery resource for proteomics. Nucleic Acids Res, 2004, 32: D497-501

62 Ikeda MD, Larkin A. ZAP70-related severe combined immunodeficiency. In: Pagon RA, Adam MP, Ardinger HH, Bird TD, Dolan CR, Fong CT, Smith RJH, Stephens K, eds. SourceGeneReviews ${ }^{\circledR}$. Seattle: University of Washington, Seattle, 2009

63 Russell SM, Johnston JA, Noguchi M, Kawamura M, Bacon CM, Friedmann M, Berg M, McVicar DW, Witthuhn BA, Silvennoinen O. Interaction of IL-2R beta and gamma c chains with Jak1 and Jak3: implications for XSCID and XCID. Science, 1994, 266: 1042-1045

64 Sebastian K, Borowski A, Kuepper M, Friedrich K. Signal transduction around thymic stromal lymphopoietin (TSLP) in atopic asthma. Cell Commun Signal, 2008, 6: 5

Open Access This article is distributed under the terms of the Creative Commons Attribution License which permits any use, distribution, and reproduction in any medium, provided the original author(s) and source are credited. 